Slavica

bruxellensia

\section{Slavica bruxellensia}

Revue polyphonique de littérature, culture et histoire

slaves

12 | 2016

Miniatures

\title{
Traduction de textes de Boris Magometovič Čipčikov
}

Traduction du russe

\section{Aleksandra Zywert et Kinga Bielerzewska}

\section{(2) OpenEdition}

Journals

Édition électronique

URL : http://journals.openedition.org/slavica/1791

DOI : 10.4000/slavica. 1791

ISSN : 2034-6395

Éditeur

Université libre de Bruxelles - ULB

Référence électronique

Aleksandra Zywert et Kinga Bielerzewska, «Traduction de textes de Boris Magometovič Čipčikov », Slavica bruxellensia [En ligne], 12 | 2016, mis en ligne le 15 novembre 2016, consulté le 19 avril 2019.

URL : http://journals.openedition.org/slavica/1791; DOI : 10.4000/slavica.1791

Ce document a été généré automatiquement le 19 avril 2019

\section{(c) $($ ) $(9)$}

Les contenus de Slavica bruxellensia sont mis à disposition selon les termes de la Licence Creative Commons Attribution - Pas d'Utilisation Commerciale - Pas de Modification 3.0 France. 


\section{Traduction de textes de Boris Magometovič Čipčikov}

Traduction du russe

Aleksandra Zywert et Kinga Bielerzewska

\section{Présentation}

Boris Magometovič Čipčikov(26 décembre 1948-21 septembre 2014), fait partie des écrivains les plus originaux de Kabardino-Balkarie, l'une des sept républiques caucasiennes autonomes faisant partie de la Fédération de Russie. Originaire d'une famille d'émigrés balkars déplacée de force vers la Kirghizie en 1944, il est né au village de Krasnaja Rečkadans, en Kirghizie. Les répressions du régime stalinien visant la population de Balkarie étant nombreuses à cette époque, son retour vers la patrie ne peut avoir lieu qu'en 1958 ; Čipčikov habitera alors à NaLčik jusqu'à la fin de sa vie.

Diplômé en histoire de l'Université de Naučik en 1978, ses premiers récits paraissent dès les années 1970 dans la presse, en russe et en balkar. En tant que membre de l'Association des Écrivains de la Fédération de Russie et secrétaire de l'organisation des Écrivains de la République Kabardino-Balkare, il mène une vie active au sein de la communauté balkare ; il est aussi instructeur de tourisme à ski. Boris Čipčikov est l'auteur de Reviens libre ( Vozvraščajsja svobodnoj, 1998), Les sourires de Dieu (Ulybaetsja Bože, 2000, écrit en balkar), La fraie des poissons au clair de lune (Nerestilis' ryby v svete lunnom, 2006).

En 2008, la maison d'édition Elbrusoid a publié, à l'occasion du soixantième anniversaire de l'auteur, un livre intitulé Nous étions voisins du Graal (My žili rjabyškom s Graalem, 2008). Dans cette publication faisant partie de la série «Les Classiques Kabardino-Balkares », on retrouve, à côté des œuvres précédentes, treize nouveaux récits, beaucoup de miniatures ainsi que la traduction d'une grande partie des récits écrits en langue balkare.

L'art d'écrire de Čipčikov se distingue par sa netteté d'expression, sa poésie du langage, sa profondeur philosophique et sa perception lyrique de l'être. L'originalité de son style, caractérisé par l'usage de procédés postmodernes ayant pour but d'exprimer une compréhension organique de la réalité, fait également partie des traits particuliers de son 
œuvre littéraire. Ses œuvres abordent les sujets principaux suivants : un nouveau sens de la vie, de l'individu et de la religion dans le contexte du pouvoir et de la faiblesse de l'homme. La conception postmoderne de Čipčikov prend ses sources dans «la mort des super-fondements »: ceux de l'homme (l'humanisme), de l'auteur (Barthes) et de Dieu (Nietzsche). En même temps, l'auteur oppose à ce paradigme thanatologique une autre conception - la foi intimidante et sans limites en la bonté de l'homme. De cette façon Čipčikov, tout en restant dans l'esthétique du postmodernisme, en repousse les frontières par son engagement en tant qu'écrivain dans les domaines artistiques et méthodologiques.

Les miniatures présentées ici n'ont jamais été publiées en français. Elles proviennent du livre Nous étions voisins du Graal (My žili rjabyškom s Graalem, 2008).

\section{Les Chats (1980)}

2 Tu rencontres un inconnu et tu te demandes : comment ne rien perdre? Et les chats, je prends, je prends et comment le leur rendre? Je ne sais pas, c'est même impossible. Il arrive parfois que tout soit mort, qu'il ne reste plus personne, seulement des montagnes, des pierres et des routes désertes... Cette créature divine monte sur ta poitrine, elle te masse avec ses pattes, elle te réchauffe et tu penses : ce n'est rien, demain tout changera, tout reviendra et toi, tu reviendras. Comment le lui payer? Avec nos méchancetés, notre cupidité, nos traîtrises... Le chat n'a besoin de rien de tout ça. Que puis-je lui offrir, moi qui ne sème rien mais qui rampe. Moi qui, des années durant, n'entendais pas le piaulement d'un petit oiseau et qui prétendais pourtant ne pas pouvoir vivre sans musique? Que puis-je donner, étant plus inutile qu'une feuille tombée au sol? Elle, au moins, elle réchauffe et nourrit la terre. Que puis-je faire, moi qui ne vois pas les étoiles et qui crache dans le feu? Que puis-je faire pour cette petite créature duveteuse, couchée sur mon lit négligé. Je ne fais que prendre d'elle. Au petit matin, tout comme elle, je m'étire sur mon lit, j'essaie, tout comme elle, de sourire à l'avant-goût du jour. Après je me lave, j'essaie, tout comme elle, de manger et, comme elle, de marcher précautionneusement sur terre. Et j'apprends d'elle cet amour. Ma dette me gêne, on ne peut pas seulement prendre tout le temps. J'attends le jour où je mettrai une chemise blanche, je me laverai avec de la rosée et j'essaierai de vivre sans m'affairer ne serait-ce qu'un jour, de me rappeler à moi-même, de me retrouver, et peut-être que là, je pourrai moi aussi offrir quelque chose à mes professeurs.

Dans chaque chat il y a un grand secret. Quand il dort, il rêve de sa patrie, l'Arabie. J'essaie d'entendre le rêve du chat... Il rêve du soleil, du désert, des dromadaires et de la chaleur. Il rêve des pyramides et des pharaons, de la Bible, du Coran et du Talmud. Il se souvient de Gilgamesh, rêve des premiers chants, des premiers sons des tam-tams. Ce tout petit être a absorbé toute l'histoire, la musique, la danse, l'architecture et tout ce torrent danse et chante en lui. Il écoute la musique des siècles écoulés et sourit dans son sommeil. D'un air maussade, il regarde la neige par la fenêtre, le poêle, la télévision, les murs blancs et froids. Il croit que c'est un cauchemar. Il ferme les yeux et retourne à la vraie vie. Après un de ces rêves de chat, Čajkovskij, Beethoven et Chopin ne paraissent plus aussi grands parce que chacun d'eux n'a entendu qu'un fragment de ce rêve. Musorgskij et Bach l'ont vu en entier. Et ils ont coupé la Terre en deux - à l'est règne Musorgskij, à l'ouest Bach, et le petit chat dort étendu sur mon lit négligé, il dort et sourit en se vautrant dans la musique des siècles passés. 


\section{Une bagatelle (1982)}

L'homme a vu dans les herbes dorées de l'année passée un fil mauve. Il s'est énervé et a dit aux autres à quel point ils étaient sales et négligents. Beaucoup d'autres paroles justes, nécessaires et méchantes grandissaient dans son for intérieur. Un autre homme vit le même fil, roulé comme une fleur et il pensa aux premières perce-neiges. Il s'est endimanché et il a couru pour annoncer aux autres l'arrivée du printemps.

Je regarde les arbres, j'entends au loin le fracas d'une chute de pierres, un garçonnet cogne une porte en fer avec un bâton. Un son désagréable mais il contient la vérité sur notre vie. Je regarde les arbres et je pense combien de choses inutiles on a dites sur nousmêmes et sur les autres. Je regarde les arbres automnaux, je les écoute, j'observe les gouttes d'eau tombant avec parcimonie, comme des points et des virgules dans les phrases des arbres. Ô combien les arbres sont-ils taciturnes et ô combien leurs paroles sont-elles importantes. Je regarde les arbres automnaux, je les écoute et je diminue, une minute après l'autre...

\section{Une salve de pissenlits (s. d.)}

4 Elle monta dans une marchroutka ${ }^{1}$ et s'installa sur le côté, il n'y avait pas d'autre place. Nous, nous entrions et elle, elle apparaissait silencieusement sans se faire remarquer. Elle était toute en taches de son, elle avait un petit nez d'oiseau, ses petites lunettes étaient rondes et vieillottes. On avait l'impression que son corps si frêle ne se composait que de tout petits os. Calmement, elle s'installa sur un petit bout de siège. Une énorme femme s'assit à côté d'elle - et elles n'étaient pas serrées. Et cela était complètement incompréhensible : est-ce le petit qui se serre contre le gros ou alors le gros qui se dissout dans le petit? Ensuite deux petites rigolotes avec le ventre à l'air se sont mises à côté et elles non plus n'étaient pas serrées. Et à travers ces taches de son et ces petits os transparents se déversait une lumière chaude, inaccessible, festive.

Et je me suis dit: si maintenant on touchait sa main, la petite lumière s'éteindrait. On ne peut pas dominer cette lumière mais il nous faut absolument nous en souvenir pour survivre. La vie ne s'accroche pas à cette femme visible mais à la lumière invisible qui émane d'elle. Et juste à ce moment-là, j'ai senti et je suis tombé amoureux de l'intellect l'intellect féminin. Non, dans le mot intellect on peut sentir une étroitesse. Oui, l'intellect et l'amour terrestre étaient des parts importantes, mais malgré tout seulement des parts de cette lumineuse infinité.

Dans mon enfance je mettais des lucioles dans une boîte parce que je voulais me réjouir à satiété de cette merveille, mais leur petite lanterne s'éteignait tout de suite parce que les lucioles, petites magiciennes, veulent briller pour tout le monde. Et c'est seulement au déclin de ma vie que j'ai compris : « On ne peut pas maitriser la lumière d'autrui, on peut seulement s'en réjouir et cette joie te remplira toi aussi de sa lumière ». Et je ne sais pas encore pourquoi cette pensée m'a effleuré l'esprit: « Le dernier envoyé de Dieu sera le Goût Absolu. Il n'y aura ni parole, ni prophétie. Sa présence silencieuse sera compréhension et plénitude pour l'homme ». Et j'ai eu une envie débordante de rouler et rouler.

Elle a arrêté la marchroutka près du Centre d'Allergologie, a doucement fermé la porte et 
elle est partie parmi les pins verts, près d'une petite tonnelle rose à la Lermontov où passaient devant les yeux les femmes de Puškin, en robes blanches et ajourées. Elle marchait parmi le rose et le vert vers le jaune clair, comme un nouveau-né vers sa maison.

Les pins grandissent en se soutenant mutuellement, en luttant pour l'étendue terrestre et l'espace céleste. Ils savent à quel point la liberté est inestimable.

Mais ils sont comme d'un autre monde, rien à voir avec toi.

Les pins d'hôpital, même s'ils grandissent très haut, restent sur terre, ce sont nos peines et souffrances qui ne les laissent pas partir. Elle marchait à côté de ces pins tristes - elle souffrait d'allergie. Elle ne pouvait avoir d'autres maladies simplement parce qu'il n'y avait pas de place pour elles dans cette lumière sans corps.

Un jeune et très fier garçon marchait sur le trottoir en portant précautionneusement sur un cintre sa douce croix si longtemps attendue, un uniforme de milicien tout neuf avec deux étoiles sur les épaulettes. Un jour de marche nous séparait de la guerre et elle était seulement à un pas de moi. J'allais à la colonie "Cimenterie", à la maison, rue de la Trompe. Il n'y a jamais eu de trompe là-bas et l'endroit faisait plutôt penser à de la ferraille. Est-ce parce qu'il était si abandonné et abîmé, va savoir?

Des jeux de lumières réfléchies dans les fenêtres à travers les buissons m'ont rappelé une tout autre histoire, longue et très étendue dans le temps et dans l'homme...

Une petite fille se mourait... sa mère en larmes... En voyant la souffrance maternelle, la petite fille, fatiguée, sourit: "Maman, ne pleure pas quand je serai morte, va dehors, regarde les pissenlits... et pense à moi ». J'étais assis sur le seuil avec mon vieux chien, le vent transportait la poussière d'une montagne creusée - du ciment - il la portait et en couvrait nos petites maisons, construites pour les ouvriers d'une usine active Dieu sait quand. Il parsemait les champs et les feuilles vertes des arbres tristes. On avait l'impression que tous, les maisons, les arbres et nous, étions complètement cimentés. Pour me sortir de cet étouffant sentiment de désespoir, je regardais le ciel mais lui aussi était comme endommagé, comme nous tous assis sous lui.

Il y a longtemps, très longtemps, loin, loin dans un champ blanc de pissenlits défleuris, avançait une petite fille en souriant, et ce sourire lointain anime notre contrée aujourd'hui.

Il faisait un peu plus chaud, une légère brise soufflait... On avait envie de voir un jour ce champ dépourvu de sens tirer une salve de pissenlits blanchis qui aiment la vie.

Et moi, j'y ai cru, je caressais la tête chaude et fatiguée de mon bon bâtard et il me regardait avec ses yeux qui comprennent... Il croyait à la même chose. Nous avons la même vie. Elle est la même pour les bi- et les quadrupèdes.

\section{NOTES}

1. Marchroutka - petit bus, moyen de transport en commun 
INDEX

Index chronologique : XXe siècle

Mots-clés : littérature balkare, littérature russe

Index géographique : Kabardino-Balkarie

\section{AUTEURS}

\section{ALEKSANDRA ZYWERT}

docteur de l'Université Adam Mickiewicz, Poznań, Pologne

\section{KINGA BIELERZEWSKA}

traductrice indépendante 